\title{
Postpartum reverse-Takotsubo from pheochromocytoma diagnosed by bedside point-of-care ultrasound: A case report
}

\author{
Jordan K. Leitch, MD, FRCPC; Anthony M.-H. Ho, MD, FRCPC, FCCP; Rene Allard, MD, FRCPC; \\ Glenio B. Mizubuti, MD, MSc \\ Department of Anesthesiology and Perioperative Medicine, Kingston Health Sciences Centre, Queen's University, \\ Kingston ON, Canada
}

\begin{abstract}
Point-of-care ultrasound is invaluable in the setting of obstetric anesthesia, where the differential diagnosis for dyspnea, hypoxemia and/or hemodynamic abnormalities is broad. This report describes a previously apparently healthy parturient with an uncomplicated pregnancy at 35-weeks gestation who underwent an emergency cesarean section under general anesthesia due to severe acute abdominal pain and fetal bradycardia. Intraoperatively, she presented with severe hypertension and tachycardia that were difficult to control and associated with ischemic ECG changes. In the immediate postoperative period, she developed retrosternal tightness and dyspnea, and a bedside point-of-care ultrasound scan revealed a grossly dilated and hypokinetic left ventricle, as well as diffuse B-lines throughout all lung fields - consistent with cardiogenic pulmonary edema. She was admitted to the intensive care unit, where she recovered over several days. Pheochromocytoma was subsequently diagnosed, and she eventually underwent uneventful elective adrenalectomy after appropriate endocrine and hemodynamic optimization.
\end{abstract}

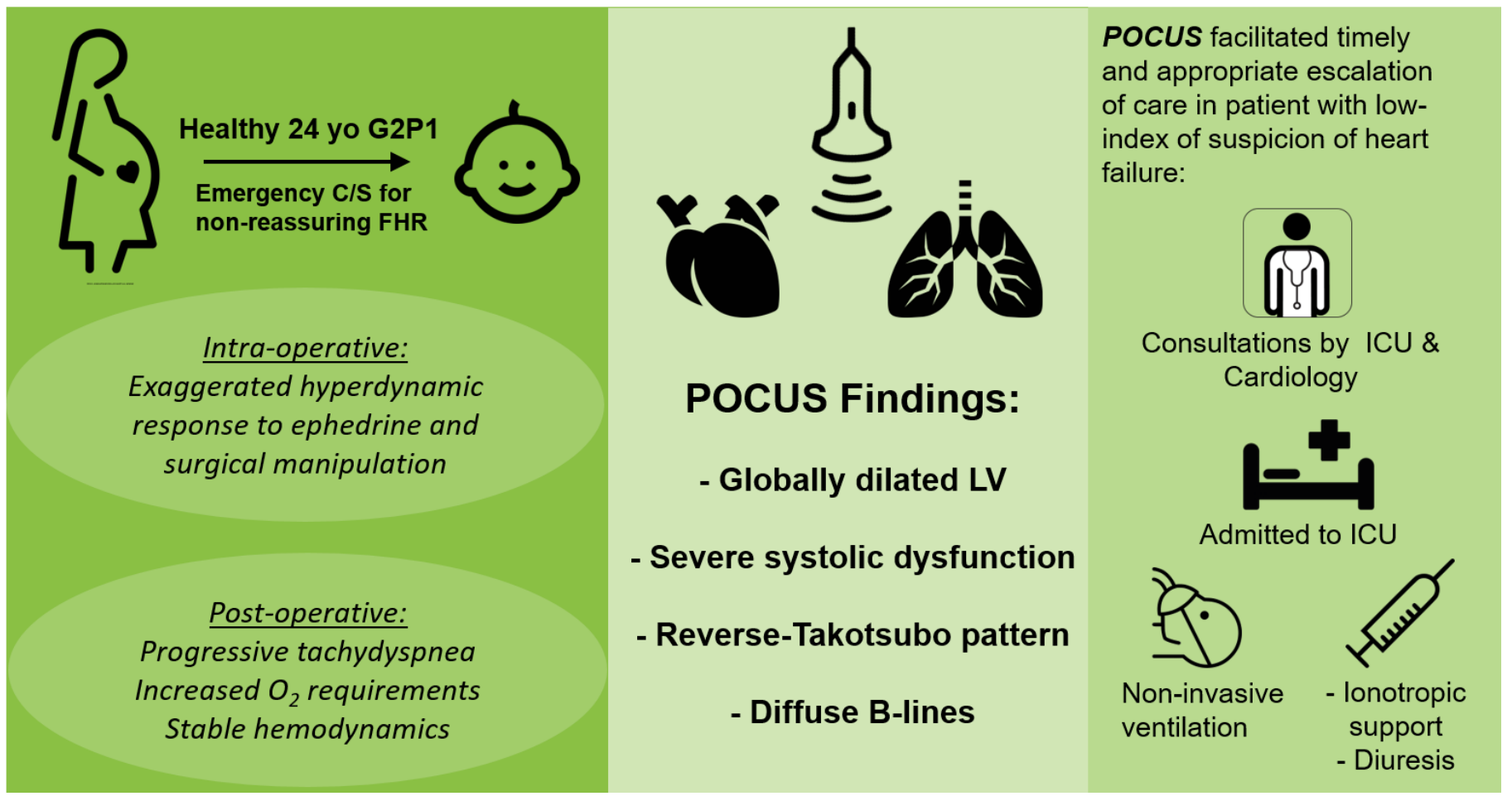

\section{Introduction}

The use of point-of-care ultrasound (POCUS) in acute care medicine has grown exponentially. Given its noninvasiveness, portability, and excellent image resolution, ultrasound (US) machines allow clinicians to quickly and serially - assess an unstable patient at the bedside, and guide management by ruling in or out important differential diagnoses which often present conflicting management goals. POCUS is invaluable in the setting of obstetric anesthesia where the differential diagnosis for dyspnea, hypoxemia and/or hemodynamic abnormalities is particularly broad - and often life-threatening [1].

We present a case in which a previously (apparently) healthy parturient with an uncomplicated pregnancy presented antepartum in a hyperdynamic circulatory state which worsened during emergency cesarean section. When she developed mild dyspnea $\left(\mathrm{SpO}_{2}=95 \%\right.$ on room air) post-partum, a POCUS examination revealed a severely hypokinetic and dilated left ventricle, which triggered a cardiology consultation and critical care 
admission. As such, POCUS facilitated the rapid diagnosis of congestive heart failure and timely management with non-invasive ventilation, inotropic support and diuresis in a patient in whom cardiogenic shock would have otherwise been substantially further down the diagnostic pathway. Furthermore, prompt identification of severe cardiomyopathy (in addition to the patient's intra-operative hemodynamic state) provided the impetus to recommend imaging and urine metanephrine testing, which confirmed the diagnosis of pheochromocytoma, and allowed for its appropriate management. Notably, these investigations were critical to distinguishing pheochromocytoma-induced cardiomyopathy from peripartum cardiomyopathy, the latter of which poses a significant risk to future pregnancies (recurrence rates: $25-100 \%$ [2]). Local ethics approval and written consent were obtained for publication of this report.

\section{Case Description}

A 24-year-old parturient G2P1 (35 weeks+2 days) was admitted with a 3-hour history of acute and rapidly progressive lower abdominal pain and non-reassuring fetal heart rate (FHR) not associated with vaginal bleeding/discharge. The patient reported an otherwise uncomplicated pregnancy and denied substance abuse. Her past medical history was unremarkable. On arrival, her blood pressure (BP) was $135 / 82 \mathrm{mmHg}$, heart rate (HR) $70 \mathrm{bpm}, \mathrm{SpO}_{2}=98 \%$, and $\mathrm{FHR} 115 \mathrm{bpm}$. Fetal movement was reportedly absent since the onset of pain. Cervical examination revealed intact membranes and no bleeding/discharge. Uterine irritability was obvious on abdominal examination, with no evidence of peritoneal irritation. Bedside US revealed an anterior placenta with no signs of abruption, and an infant in cephalic presentation with now sustained bradycardia (85 bpm). The patient was immediately transferred to the operating room (OR) due to suspicion of uterine rupture and/or placental abruption.

In the OR, ECG, $\mathrm{SpO}_{2}$, and non-invasive $\mathrm{BP}$ were applied, and general anesthesia (GA) was induced, followed by tracheal intubation. Anesthesia was maintained with sevoflurane $\left(\mathrm{Et}_{\mathrm{SEV}} \sim 2 \%\right)$. Given the possibility of ongoing hypovolemia, crystalloids were rapidly infused through 2 peripheral $18 \mathrm{G}$ intravenous lines and ephedrine $10 \mathrm{mg}$ was administered upon induction. This triggered an unusual and disproportionate hypertensive $(215 / 110 \mathrm{mmHg})$ response associated with ECG changes (ST depression and $\mathrm{T}$ wave inversion) which was managed with propofol $(50 \mathrm{mg})$. A Pfannenstiel incision was performed and the infant delivered without complication. Given persistent hypertension (systolic BP $170-200 \mathrm{mmHg}$ ) and tachycardia (130 bpm), incremental doses of fentanyl and morphine (total of $250 \mathrm{mcg}$ and $10 \mathrm{mg}$, respectively) were administered on the assumption that the hemodynamics were pain-related, with negligible effect. No free fluid/ blood was noted upon entry into the abdomen, and the uterus appeared normal (no evidence of Couvelaire uterus). Amniotic fluid was clear, and the placenta did not show evidence of abruption. The placenta appeared smaller than normal consistent with chronic ischemia, which also raised the possibility of acute uterine ischemia as a cause of her abdominal pain. Urinary bladder, ovaries and fallopian tubes were intact. Given the lack of an obvious reason to explain the patient's acute and severe preoperative abdominal pain, general surgery was consulted intraoperatively; however, no intra-abdominal pathology was identified. Notably, intraoperative palpation of the patient's right upper abdominal quadrant triggered severe hypertension $(220 / 100 \mathrm{mmHg})$ and tachycardia (135 bpm). This was treated with hydralazine $(40 \mathrm{mg})$, and prompted the anesthesia team to arrange for phentolamine as undiagnosed pheochromocytoma was now suspected. Estimated blood loss was $500-700 \mathrm{~mL}$, and the patient received a $1500 \mathrm{~mL}$ of crystalloid. Upon completion of surgery and tracheal extubation, the patient was transferred to the post-anesthetic care unit in stable condition $\left(\mathrm{BP}=142 / 90 \mathrm{mmHg}, \mathrm{HR}=120 \mathrm{bpm}, \mathrm{SpO}_{2}=\right.$ $97 \%$ on room air).

Shortly after, the patient complained of progressive retrosternal tightness and shortness of breath. She was tachypneic $(26 \mathrm{rpm})$ and diaphoretic, with bilateral basal crackles. Vital signs were BP $=145 / 89 \mathrm{mmHg}, \mathrm{HR}=110$ bpm, and $\mathrm{SpO}_{2}=95 \%$ on oxygen $(15 \mathrm{~L} / \mathrm{min})$ through a non-rebreather facial mask. Blood work revealed an elevated CK (262 U/L) and troponin (1111 ng/L). Toxicology profile was negative and the remaining blood tests (CBC, electrolytes, preeclampsia panel, TSH and $\mathrm{PTT} / \mathrm{INR}$ ) were within normal range. A bedside POCUS revealed a globally dilated left ventricle with severe systolic dysfunction and wall motion abnormalities consistent with reverse-Takotsubo pattern (basal and mid -segment hypokinesis/akinesis and apical segment hyperkinesis), normal right ventricular function, no pericardial effusion, severe mitral regurgitation, and mildto-moderate tricuspid regurgitation (Figure 1, online Videos S1-5). These findings were readily apparent to the operators (JKL and GBM) and later confirmed by a formal transthoracic echocardiogram (without contrast) performed by a certified echocardiographer. Bilateral multiple B-lines were identified on lung US. At this point, BiPAP, dobutamine infusion and furosemide $40 \mathrm{mg}$ were administered upon transferring the patient to the ICU. The differential diagnosis included pheochromocytoma, thyrotoxicosis, and pre-existing viral or peripartum cardiomyopathy. Particularly, pheochromocytoma was suspected on the basis of the disproportionate 


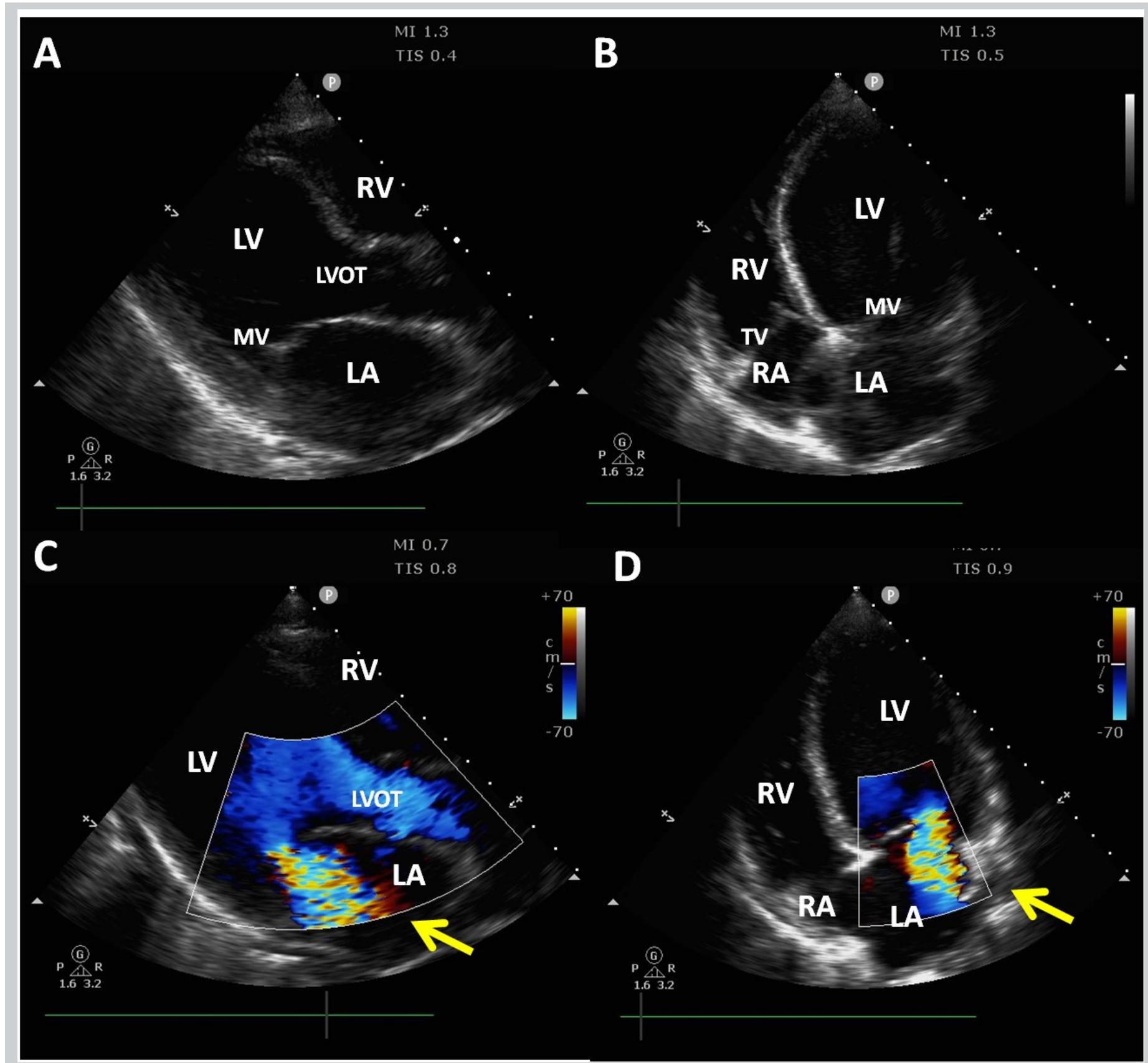

Figure 1. Parasternal long axis (A) and apical 4-chamber (B) views showing a dilated left ventricle (LV). Similar views are shown in panels $(C)$ and $(D)$, respectively, with color Doppler demonstrating significant mitral regurgitation (yellow arrow). RV = Right ventricle; LVOT = Left ventricular outflow track; RA = Right atrium; MV = Mitral valve; TV = Tricuspid valve.

hyperdynamic response to both ephedrine and direct palpation of the right upper abdominal quadrant. (Ephedrine is an indirect acting vasopressor that stimulates the release of endogenous catecholamines which makes it relatively contraindicated in pheochromocytoma due to potential for massive release of tumoral catecholamines and subsequent hemodynamic instability. Similarly, direct mechanical mobilization (e.g., palpation) of the tumor may result in an extreme hyperdynamic response from a similar (i.e., tumoral catecholamine release) mechanism). On further investigation, a contrasted computed tomography scan revealed a $5 \times 4 \times 4 \mathrm{~cm}$ right adrenal gland mass and the diagnosis of pheochromocytoma was subsequently confirmed with urine metanephrine testing. Later in the year, after optimization by a multidisciplinary team, this patient underwent laparoscopic right adrenalectomy uneventfully. At the time of this writing, both patient and infant are in good health and thriving. 


\section{Discussion}

Coexistence of pregnancy and pheochromocytoma is rare. Only $1 / 5$ of cases are identified in the peripartum period, typically as a result of complications [3]. In our case, the pheochromocytoma manifested with the onset of abdominal pain/labor, and the diagnosis was facilitated by the use of POCUS.

Heart disease is a major cause of maternal morbidity and mortality $[2,4]$. While obstetric critical illness frequently presents on the background of good health and significant physiologic reserve, the obstetric population is evolving to include advanced maternal age, morbid obesity, and heart disease [1]. With this reality, obstetric guidelines now emphasize the importance of the skill set to diagnose and manage heart failure in pregnancy, with emphasis placed on early identification of patients who require intervention $[2,4]$. Dyspnea, a common symptom in the peripartum period, can be benign and self-resolving (e.g., atelectasis, abdominal pain) or an early warning of critical illness (e.g., pulmonary embolism, hemorrhage, heart failure). In this setting, POCUS is an invaluable tool that allows clinicians to narrow down a long list of potential diagnoses (and, importantly, to distinguish between cardiac and respiratory etiologies), facilitating early management before frank clinical decompensation $[1,5,6]$. POCUS consists of a guideline-recommended diagnostic modality which allows the treating physician to not only serially assess an unstable patient, but also to guide the response to administered therapies. Specifically, transthoracic echocardiography-guided management of hemodynamic instability adheres to the highest level of evidence according to the American and European societies governing the management of heart failure $[5,7]$.

Parturients are well-suited to POCUS examination. The lateral and anterior displacement of the heart by the gravid uterus, and the known benefits of aortocaval compression relief in the left lateral position may facilitate image acquisition (except for subcostal views). Indeed, Jain and colleagues found that $93 \%$ of term parturients examined with echocardiography had image quality rated as "good" or "perfect" in both the parasternal and apical views. In addition, body mass index (BMI) did not significantly change the quality of images obtained [8].

Dennis and colleagues published the rapid obstetric screening echocardiography (ROSE) protocol to guide anesthesiologists in assessing cardiac function as the etiology of dyspnea or hemodynamic instability, and it serves as a pragmatic tool guiding cardiac assessment of parturients [1]. The ROSE protocol can be supplemented with rapid bedside lung US in emergency (BLUE) protocol [9] to enhance assessment of peripartum dyspnea. The BLUE protocol provides valuable information about pulmonary pathology [9] and is, therefore, of importance during pregnancy in which dyspnea is a common complaint and avoidance of ionizing radiation is desirable. Specifically, the analysis of three major lung ultrasound "signatures" (A-lines, B-lines and lung-sliding) offers superior sensitivity $(97 \%)$ and specificity $(95 \%)$ in the diagnosis of pulmonary edema $[9,10]$. Our patient presented a B-line profile that corroborated the diagnosis of cardiogenic dyspnea [11,12] thereby directing our decision-making to include non-invasive positive pressure ventilation, inotropy and diuresis as part of the treatment.

While several anesthesia residency programs require POCUS training, sufficient infrastructure is not yet ubiquitous to support the role of this technology in our field. Beyond maintaining competency with bedside practice and workshop attendance [13], it is important to have secure digital archives of scans from actual cases to review amongst the clinician group in a systematic fashion for educational purposes [14]. Finally, it is important to recruit parturients into research studies [15] using emerging modalities such as POCUS to develop this body of literature, thereby allowing this group of patients to benefit from ongoing technical advances and knowledge.

Anesthesiologists play a central role in the management of critically ill obstetric patients, and clinicians skilled in POCUS are able to quickly and accurately diagnose lifethreatening pathology and adjust management accordingly. Indeed, the current case serves as a practical example of how POCUS can influence the decision-making and directly (and positively) affect clinical management in a critically ill parturient.

\section{Declaration of patient consent}

The authors certify that they have obtained all appropriate patient consent forms. In the form the patient has given her consent for her images and other clinical information to be reported in the journal. The patient understands that her name and initials will not be published and due efforts will be made to conceal her identity, but anonymity cannot be guaranteed.

\section{References}

1. Dennis AT. Transthoracic echocardiography in obstetric anaesthesia and obstetric critical illness. Int J Obstet Anesth 2011;20(2):160-8.

2. Dennis AT. Heart failure in pregnant women: Is it peripartum cardiomyopathy? Anesth Analg 2015;120(3):638-43.

3. Biggar MA, Lennard TWJ. Systematic review of phaeochromocytoma in pregnancy. Br J Surg 2013;100(2):182-90.

4. Cantwell R, Clutton-Brock T, Cooper G, et al. Saving Mothers' Lives: Reviewing maternal deaths to make motherhood safer: 2006-2008. The Eighth Report of the Confidential Enquiries into Maternal Deaths in the United Kingdom. BJOG 2011. 


\section{1 | POCUS J | NOV 2020 vol. 05 iss. 02}

5. Regitz-Zagrosek V, Blomstrom Lundqvist C, Borghi C, et al. ESC Guidelines on the management of cardiovascular diseases during pregnancy. Eur Heart J 2011;32(24):3147-97.

6. Dennis A, Stenson A. The use of transthoracic echocardiography in postpartum hypotension. Anesth Analg 2012;115(5):1033-7.

7. Yancy CW, Jessup M, Bozkurt B, et al. 2013 ACCF/AHA Guideline for the Management of Heart Failure. J Am Coll Cardiol 2013.

8. Jain D, Grejs AM, Bhavsar R, et al. Focused cardiac ultrasound is feasible in parturients; a prospective observational study. Acta Anaesthesiol Scand 2017.

9. Lichtenstein DA, Mezière GA. Relevance of lung ultrasound in the diagnosis of acute respiratory failure the BLUE protocol. Chest 2008.

10. Lichtenstein DA. BLUE-Protocol and FALLS-Protocol: Two applications of lung ultrasound in the critically ill. Chest 2015.

11. Zieleskiewicz L, Lagier D, Contargyris $C$, et al. Lung ultrasoundguided management of acute breathlessness during pregnancy. Anaesthesia 2013.

12. Ambrozic J, Brzan Simenc G, Prokselj K, et al. Lung and cardiac ultrasound for hemodynamic monitoring of patients with severe preeclampsia. Ultrasound Obstet Gynecol 2017;49(1):104-9.

13. Price S, Via G, Sloth E, et al. Echocardiography practice, training and accreditation in the intensive care: Document for the World Interactive Network Focused on Critical Ultrasound (WINFOCUS). Cardiovasc Ultrasound 2008.

14. Evangelista A, Flachskampf $F$, Lancellotti $P$, et al. European Association of Echocardiography recommendations for standardization of performance, digital storage and reporting of echocardiographic studies. Eur J Echocardiogr 2008.

15. Goldkind SF, Sahin L, Gallauresi B. Enrolling pregnant women in research - Lessons from the H1N1 influenza pandemic. N Engl J Med 2010 . 